\title{
Endoscopic features of submucosal deeply invasive colorectal cancer with NBI characteristics
}

\section{S Saito et al. Endoscopic images of early colorectal cancer}

\author{
Shoichi Saito ${ }^{1}$ Hisao Tajiri ${ }^{1,2} \cdot$ Masahiro Ikegami $^{3}$
}

Received: 30 October 2015/Accepted: 30 October 2015/Published online: 11 December 2015

(C) Japanese Society of Gastroenterology 2015

\begin{abstract}
In this review, we discuss the features of conventional endoscopy, magnified endoscopy involving image enhanced endoscopy and endoscopic ultrasonography (EUS) using illustrations for submucosal deeply invasive colorectal cancer (SM-Ca). First, the typical features of SM-Ca were observed, including fold convergence, stiffness, depression (ulceration) and elevated lesions in depressed areas. Magnified endoscopic findings using NBI showed dilated, irregularly shaped micro-capillary vessels. In addition, $V_{I}$ and $V_{N}$ pits were clearly visible using crystal violet staining. In contrast, using EUS, at the third layer we found a layer that was thin compared to the surrounding normal mucosa, which suggested the existence of SM-Ca.
\end{abstract}

Keywords Submucosal invasive cancer - Conventional endoscopy $\cdot$ Magnified endoscopy $\cdot$ Narrow band imaging (NBI) - Autofluorescence imaging (AFI) ·

Chromoendoscopy · Endoscopic ultrasonography (EUS) · High-frequency ultrasound probe (HFUP)

Shoichi Saito

ssaito@jikei.ac.jp

1 Department of Endoscopy, The Jikei University School of Medicine, 3-25-8, Nishi-shinbashi Minato-Ku, Tokyo 105-8461, Japan

2 Division of Gastroenterology and Hepatology, Department of Internal Medicine, The Jikei University School of Medicine, Tokyo, Japan

3 Department of Pathology, The Jikei University School of Medicine, Tokyo, Japan

\section{Introduction}

Endoscopic images of early colon cancer are usually obtained from one of two types of endoscopies: conventional endoscopy or magnified endoscopy. The relationship between the two methods can be compared to two wheels on a cart. It is important to use both methods, conventional and magnified endoscopy, to increase the accuracy of preoperative diagnosis for the depth of invasion and develop a treatment strategy $[1,2]$. Above all, it is critical to make a precise diagnosis of submucosal deeply invasive cancer (SM-Ca) and decide which strategy, endoscopic treatment or laparoscopic surgery, should be employed. In this review, we will provide an overview of the basics of endoscopic imaging and its usefulness, as well as the actual characteristic findings of SM-Ca by endoscopy.

\section{Features of endoscopic images of SM-Ca}

\section{Conventional endoscopic imaging (Fig. 1; Table 1)}

A characteristic finding suggestive of SM-Ca observed by conventional imaging is the extension of the bowel lumen caused by inflated air. In other words, when the following findings suggestive of SM-Ca are observed, an extension of bowel lumen caused by the inflated air should be considered poor. Moreover, it is unknown where this characteristic finding will be observed, i.e., distal, medial, or proximal from the lesion.

The Japanese Guideline for Colorectal Cancer [1] has listed the following endoscopic findings as diagnostic indicators of SM-Ca: expansive appearance, erosion/ulceration, fold convergence, and deformation/stiffness. Saitoh et al., who led the "indication for endoscopic 
Fig. 1 Features of endoscopic images of SM-Ca. a,

b Expansive appearance, c, d erosion/ulceration, $\mathbf{e}, \mathbf{f}$ fold convergent, $\mathbf{g}$, h stiffness, $\mathbf{i}$, $\mathbf{j}$ elevated lesion in a depressed area
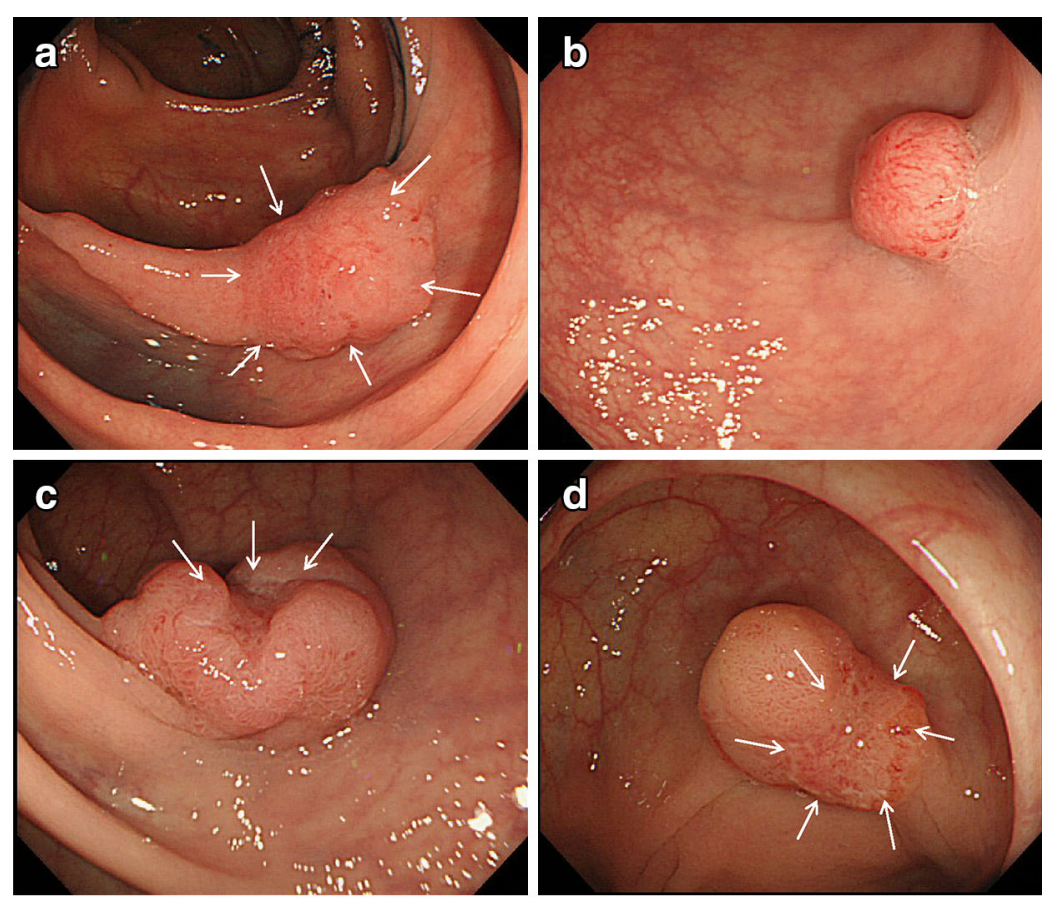

d
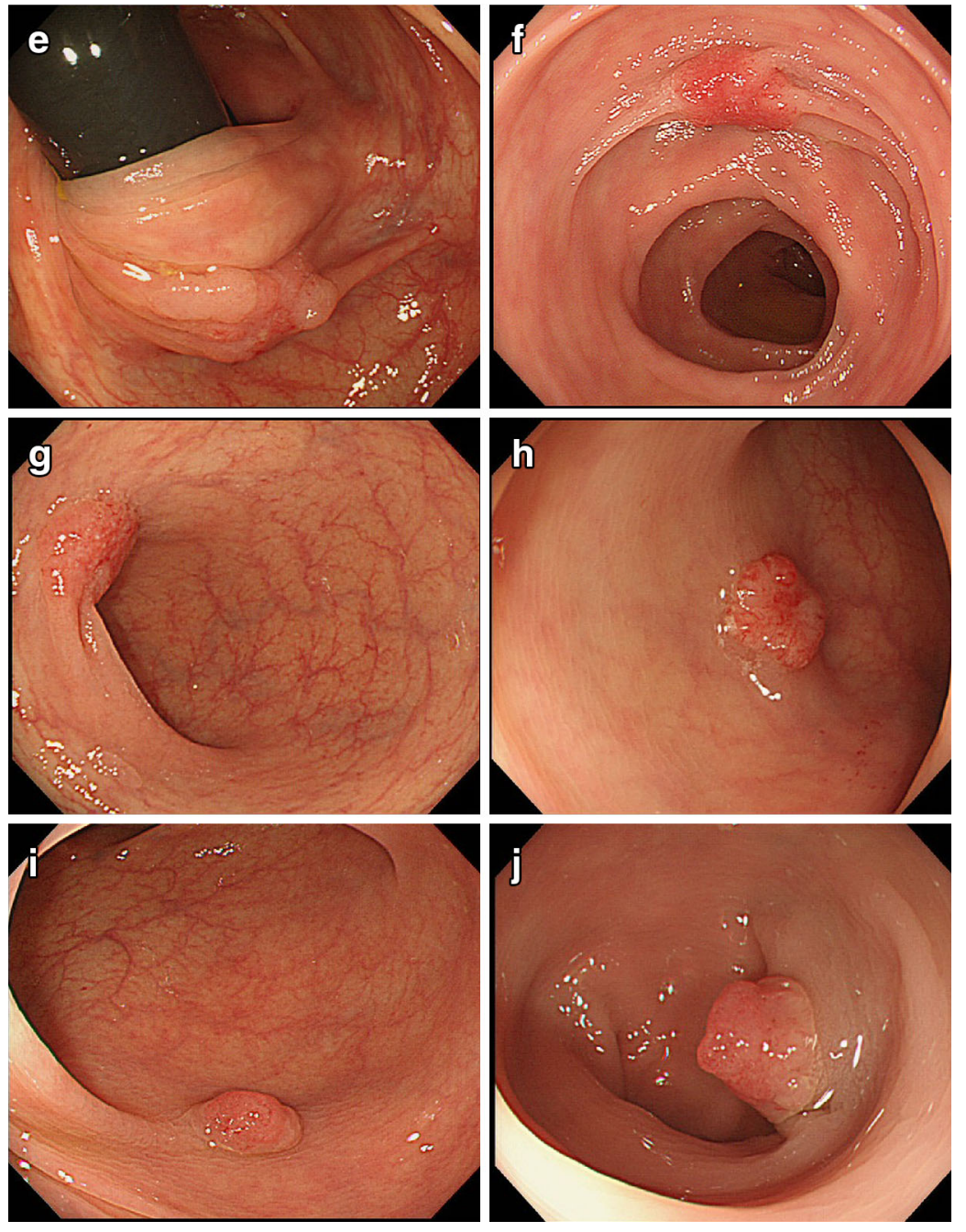
Table 1 Frequency of each feature of the endoscopic images for SM-Ca

\begin{tabular}{|c|c|c|c|c|c|}
\hline & Expansive & Erosion/ulceration & Fold convergent & Stiffness & Elevation in depression \\
\hline Kawano (3) $P$ type & $66.2 \%(31.6 \%)$ & $9.4 \%(10.0 \%)$ & & $35.5 \%(0 \%)$ & \\
\hline$S$ type & $78.9 \%(23.3 \%)$ & $3.9 \%(0 \%)$ & & $44.8 \%(13.2 \%)$ & \\
\hline Our facility $P$ type & $71.1 \%(0 \%)$ & $55.6 \%(70.0 \%)$ & $24.4 \%(0 \%)$ & $17.8 \%(0 \%)$ & \\
\hline$S$ type & $80.9 \%(33.3 \%)$ & $29.8 \%(33.3 \%)$ & $64.9 \%(16.7 \%)$ & $80.7 \%(16.7 \%)$ & \\
\hline Saitou (2) $P$ type & 0.0369 & 0.0129 & 0.0111 & 0.0004 & \\
\hline$S$ type & $<0.0001$ & 0.0458 & 0.0087 & 0.0052 & 0.0062 \\
\hline Wan (4) any type & $25.0 \%(0 \%)$ & $4.5 \%(0 \%)$ & $8.3 \%(0 \%)$ & & \\
\hline
\end{tabular}

$\%$ Indicates frequency of slightly submucosal invasive cancers

$P$ type protruded type, $S$ type superficial type

treatment" project study group in the Japanese Society for Cancer of the Colon and Rectum, conducted a multicenter prospective study. They analyzed conventional endoscopic findings, including dye-spraying findings in which the invasion into the SM layer appears deeper than $1000 \mu \mathrm{m}$ in the two types of SM-Ca, i.e., the protruded type and the superficial type. They also uncovered significant endoscopic findings for an invasion deeper than $1000 \mu \mathrm{m}$ [3] regarding each of the following groups: expansive appearance, endoscopic stiffness, depressed area, rough mucosa and a convergent fold in the protruded type SMCa. Furthermore, there was an expansive appearance, endoscopic stiffness, a depressed area, an elevated lesion in the depressed area, a rough surface, rough mucosa with reddish change, a converged fold, a convergent fold, a marked elevated lesion, no deformation with inflated air and easy bleeding in the superficial type of SM-Ca.

Kawano et al. reported that a diagnosis of cSM deeper invasion cancer could be made if any one of the following four indicators were present: an obviously deeply depressed area, an expansive appearance, an SMT-like elevated lesion, and poor distension by inflation inside the bowel at normal areas, as were observed in the above-mentioned findings [4].

Table 1 shows the characteristic findings for SM-Ca that were obtained in our study: (1) expansive appearance (Fig. 1a, b), (2) erosion/ulceration of the surface, (3) fold convergence (Fig. 1e, f), (4) wall stiffness (Fig. 1g, h), and (5) elevated lesions in the depressed area (Fig. 1i, j).

Those that satisfied any one of the above-mentioned findings accounted for approximately $80 \%$ in the case of SM-Ca, but were not limited to their morphological, macroscopic appearance. Of note, some facilities combined the third and fourth criteria listed above as a poorly distended tumor lesion by inflated air [5].

\section{Magnified endoscopic imaging (Tables 2, 3)}

It is necessary to conduct magnified endoscopic imaging after conventional endoscopy to achieve better diagnostic accuracy. Magnified endoscopic imaging is generally classified into two methods: the first method involves observing the vascular pattern and the superficial structure of the surface of the lesion using a narrow band imaging (NBI) system; the second method involves a pit pattern diagnosis using dye-spray staining.

\section{Discussion based on the vascular pattern (Table 2)}

Based on the degree of dilatation of the capillary blood vessels and the appearance of the surface pattern revealed by the NBI (ME-NBI), there are reports from four facilities in Japan, including the NBI classification, which is recommended by our study (Table 2) [6-11]. The classifications listed by all four facilities suggest that the vascular pattern and histologic type/invasion depth of lesions are correlated. In other words, if a pit-like appearance, as seen via indirect observation of the orifices of crypts, is observed instead of the dilatation of a micro-capillary, it is most likely a non-neoplastic, hyperplastic polyp (HP). If mild vasodilatation is observed, it is likely an intra-epithelial mucosal lesion mainly consisting of a tubular adenoma. When the vasodilatation becomes noteworthy and an irregular surface pattern appears, SM-Ca is strongly suspected rather than intra-mucosal cancer [12, 13]. As is shown in Table 2, the lesions indicated for endoscopic resection and those indicated for surgical resection are similarly included in the following classifications: Sano's classification-IIIA (5.5\%), Hiroshima classification-C2 (61.9\%), Jikei classification-3I (47.8\%), and Showa classification-irregular pattern $(91.3 \%)$. In these cases, a pit pattern observation using crystal violet staining is required for further examination. However, when the blood vessel pattern and surface pattern has disappeared from the tumor area and cannot be observed, SM-Ca should be considered and an immediate surgical resection is indicated. Among these cases, the incidence of SM-Ca is remarkably high: $71.8 \%$ in Sano's classification-IIIB, $93.5 \%$ in Hiroshima classification-C3, $93.6 \%$ in Jikei 
Table 2 NBI classification including the frequency of SM-Ca

\begin{tabular}{|c|c|c|}
\hline Endoscopic findings & Classification & Frequency \\
\hline $\begin{array}{l}\text { Capillary dilatation: }(-) \\
\text { Surface pattern: reg. }\end{array}$ & $\begin{array}{l}\text { Sano's classification (6): CP type I } \\
\text { Hiroshima classification (7): type A } \\
\text { Showa classification (8): Faint } \\
\text { Jikei classification (9): type } 1 \\
\text { NICE classification (10-11): type } 1\end{array}$ & $\begin{array}{l}\text { Sano's classification: } 0 \% \\
\text { Hiroshima classification: } 0 \% \\
\text { Showa classification: } 0 \% \\
\text { Jikei classification: } 0 \% \\
\text { NICE classification: } 0 \%\end{array}$ \\
\hline $\begin{array}{l}\text { Capillary dilatation: }(+) \\
\text { Surface pattern: reg. }\end{array}$ & $\begin{array}{l}\text { Sano's classification: CP type II } \\
\text { Hiroshima classification: type B } \\
\text { Showa classification: network } \\
\text { Jikei classification: type } 2 \\
\text { NICE classification: type } 2\end{array}$ & $\begin{array}{l}\text { Sano's classification: } 0 \% \\
\text { Hiroshima classification: } 0 \% \\
\text { Showa classification: } 0 \% \\
\text { Jikei classification: } 2.3 \% \\
\text { NICE classification: } 6.7 \%\end{array}$ \\
\hline $\begin{array}{l}\text { Capillary dilatation: }(++) \\
\text { Surface pattern: reg. }\end{array}$ & $\begin{array}{l}\text { Sano's classification: CP type II } \\
\text { Hiroshima classification: type } \mathrm{C} 1 \\
\text { Showa classification: dense } \\
\text { Jikei classification: type } 3 \mathrm{~V} \\
\text { NICE classification: type } 2\end{array}$ & $\begin{array}{l}\text { Sano's classification: } 0 \% \\
\text { Hiroshima classification: } 3.7 \% \\
\text { Showa classification: } 0 \% \\
\text { Jikei classification: } 6.0 \% \\
\text { NICE classification: } 6.7 \%\end{array}$ \\
\hline $\begin{array}{l}\text { Capillary dilatation: }(++) \\
\text { Surface pattern: irreg. }\end{array}$ & $\begin{array}{l}\text { Sano's classification: CP type IIIA } \\
\text { Hiroshima classification: Type C2 } \\
\text { Showa classification: Irregular } \\
\text { Jikei classification: type 3I } \\
\text { NICE classification: type } 2\end{array}$ & $\begin{array}{l}\text { Sano's classification: } 5.5 \%(11) \\
\text { Hiroshima classification: } 61.9 \%(12) \\
\text { Showa classification: } 91.3 \% \\
\text { Jikei classification: } 47.8 \% \\
\text { NICE classification: } 6.7 \%\end{array}$ \\
\hline $\begin{array}{l}\text { Capillary vessels: }(-) \\
\text { Surface pattern: }(-)\end{array}$ & $\begin{array}{l}\text { Sano's classification: CP type IIIB } \\
\text { Hiroshima classification: Type C3 } \\
\text { Showa classification: Sparse } \\
\text { Jikei classification: type } 4 \\
\text { NICE classification: type } 3\end{array}$ & $\begin{array}{l}\text { Sano's classification: } 71.8 \% \\
\text { Hiroshima classification: } 93.5 \% \\
\text { Showa classification: } 82.6 \% \\
\text { Jikei classification: } 93.6 \% \\
\text { NICE classification: } 93.5 \%\end{array}$ \\
\hline
\end{tabular}

classification-4, and $82.6 \%$ in Showa classification-Sparse pattern. Based on the these findings, it seems appropriate to use ME-NBI as a means of sorting out the appropriate treatment methods, which, in this case, would be endoscopic treatment or surgical treatment for a pit pattern observation. However, ME-NBI is slightly inferior to pit pattern diagnosis in terms of accuracy, and an almost similar outcome can be obtained [14]. The current problem is that NBI classification varies from one facility to another, which is why a unified classification using magnified observation is in development in Japan [15].
Also, the observation of white opaque substance (WOS) is recommended using ME-NBI by Kawasaki et al. [16]. This finding is suggested to closely relate to the depth of invasion into SM layer as an optical marker.

\section{Discussion based on the pit pattern (Table 3)}

There are two methods for pit pattern observation of SM$\mathrm{Ca}$ : the dye spraying method using indigo carmine spraying, and crystal violet staining. A discussion regarding pit pattern observation has long indicated the use of Kudo and 


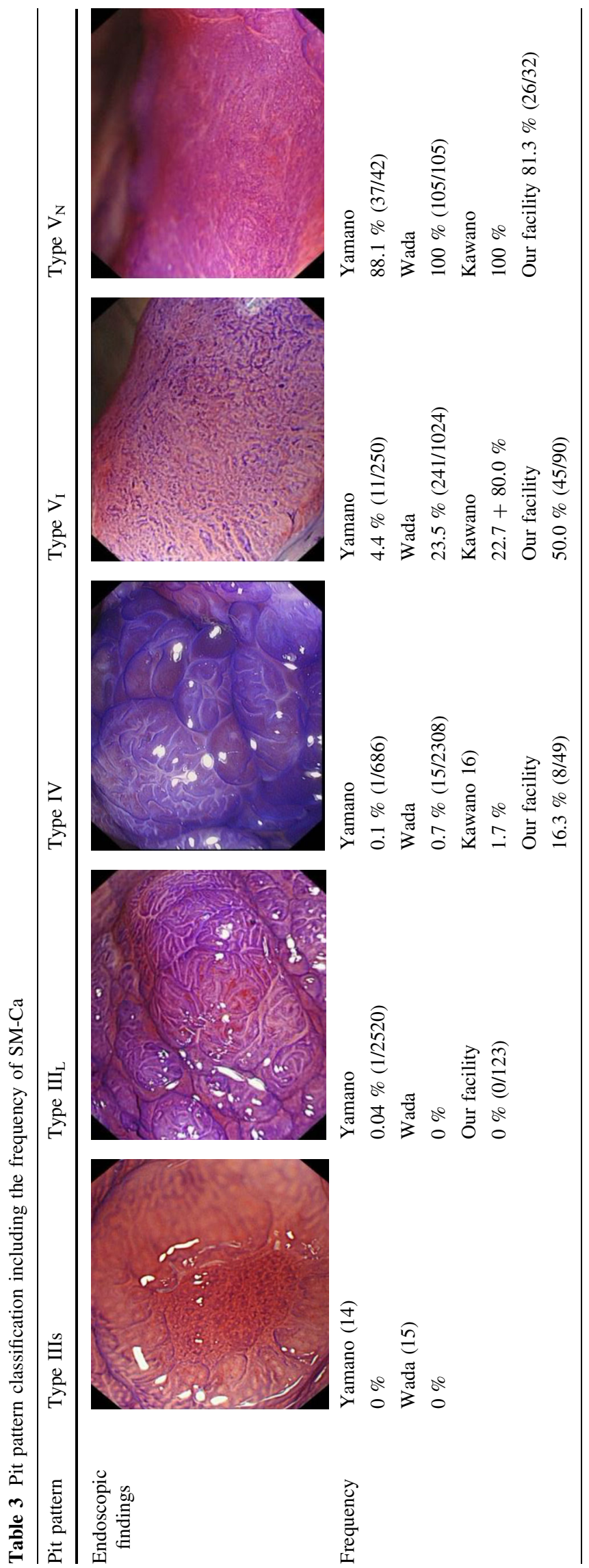

Tsuruta's classification, and therefore, can almost be considered to be an established diagnostic method in Japan.

Reports from each facility [17-19], seem to suggest no SM$\mathrm{Ca}$ involvement in lesions with type III and IV pit patterns.

For this reason, the type $\mathrm{V}$ pit pattern is mainly used to identify SM-Ca in this study. The type $\mathrm{V}$ pit is classified into two subgroups: type $\mathrm{V}_{\mathrm{I}}$ (I: Irregular) pit and type $\mathrm{V}_{\mathrm{N}}(\mathrm{N}$ : Non-structure) pit. The type $V_{I}$ pit pattern is treated with endoscopy, while the type $\mathrm{V}_{\mathrm{N}}$ pit pattern is treated with surgical re-sectioning. Yamano et al. and Wada et al. reported the incidence of SM-Ca from the type $\mathrm{V}_{\mathrm{I}}$ pit pattern as 4.4 and $23.5 \%$, respectively. Kawano et al. roughly classified the type $\mathrm{V}_{\mathrm{I}}$ pit pattern into the regular type and irregular type, and reported that each type accounts for 22.7 and $80.0 \%$, respectively; in our study, each type accounted for $50.0 \%$. Therefore, it seems that many cases, including cases classified at the regular type by Kawano et al., will utilize endoscopic treatment. The reason for the high incidence rate in our study can be attributed to the fact that we did not perform magnifying endoscopy using crystal violet staining for lesions that were obviously indicated for endoscopic treatment; instead, we only selected lesions that were difficult to judge between treatment with endoscopy or surgical resection. We think that a selection bias was present. As for the type $\mathrm{V}_{\mathrm{N}}$ pit, Yamano et al., Wada et al. and Kawano et al. reported that the incidence rate was 88.1, 100, and $100 \%$, respectively; in our study, the incidence rate was $81.3 \%$. Regardless of the method used, we think that a surgical resection can be selected for SM-Ca without hesitation, because it is a very frequent event.

\section{Autofluorescence endoscopy (AFE)}

AFE is also useful method to differentiate from non-neoplastic lesion or intraepithelial neoplasia. AFE is classified into image enhanced endoscopy (IEE) as an NBI method. We reported that autofluorescene imaging (AFI) is well related to the histological grading and depth of invasion [20-23]. Especially, the magenta color is strongly revealed as different from the surrounding normal mucosa in SM-Ca $[22,23]$.

\section{Endoscopic ultrasonography (EUS) (Fig. 2)}

The instruments of endoscopic ultrasonography (EUS) used for colorectal cancers are the echo-colonoscope and high-frequency ultrasound probe (HFUP). Because HFUP is often used in most facilities [24, 25], we will outline the diagnostic method of HFUP in this section.

We think there are some limitations in observations using EUS compared to conventional endoscopy. First, water-immersion endoscopy is sometimes difficult to use because of the 

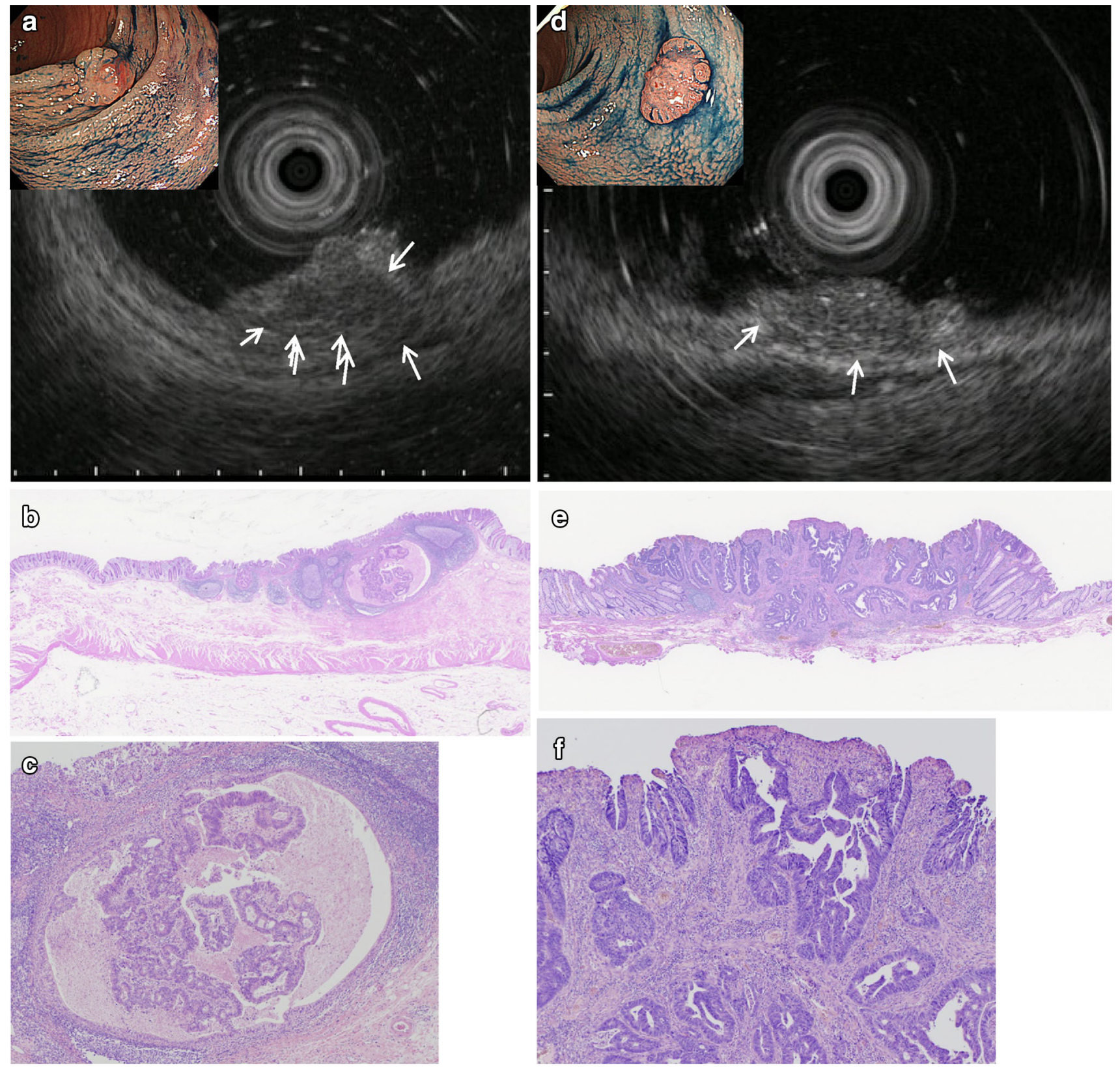

Fig. 2 Features of endoscopic images of EUS for SM-Ca. a EUS low echoic mass revealed SM deep invasion with marked lymphocyte infiltration (white arrow). b HE staining, whole specimen of deepest invasion. $\mathbf{c}$ High power view of a well-differentiated adenocarcinoma

with mucous lake. d EUS low echoic mass revealed SM deep invasion (white arrow). e HE staining, whole specimen of the deepest invasion. f High power view of a well differentiated adenocarcinoma

flexure at the bowel. In this regard, Hamamoto et al. [26], Kobayashi et al. [27] and Saito et al. [28] reported the success rate of visualization as 86,89 , and $88.9 \%$, respectively. Second, the success rate of visualization is thought to vary depending on the macroscopic appearance of the lesions [29]. Therefore, it is often difficult to visualize the protruded type, because of the attenuation at the deep portion in the lesion. Hamamoto et al. reported the accuracy rate of the protruded type as $83 \%$ and the superficial type as $93 \%$, whereas Saito et al. reported the rate as 81.4 and $92.0 \%$, respectively. In

addition, Kobayashi et al. reported the accuracy rate as $81 \%$ in both types. Although the accuracy rate is high at all facilities, differentiation from the intra-epithelial mucosal lesion might be difficult depending on the lesions, which are invaded in the shallow SM layer. Kobayashi et al. defined the standard for the diagnosis of SM deeply invasive cancers using EUS as "those with the extensive narrowing and disruption in the third layer and without any changes in the fourth layer." However, attention should be paid to some lesions in which hyperplasia of the lymphoid follicle of the submucosal layer can be 
misdiagnosed or inaccurately interpreted as an SM invasion (Fig. 2a-c). In this regard, several reports further mentioned that improvements to the diagnosis system using three-dimensional EUS (3D-EUS), in addition to its contribution to improving the accuracy of diagnosis, should be greatly expected [30].

\section{Compliance with Ethical Standards}

Conflicts of Interest: Shoichi Saito, Hisao Tajiri and Masahiro Ikegami declare that they have no conflict of interest.

\section{References}

1. Japanese Society for Cancer of the Colon and Rectum (ed.). JSCCR guideline 2014 for the treatment of colorectal cancer. Tokyo: Kanehara; 2014 (in Japanese).

2. Tanaka S, Kashida H, Saito Y, et al. JGES guideline for colorectal endoscopic submucosal dissection/endoscopic mucosal resection. Dig Endosc. 2015;27(4):417-34.

3. Saitoh Y, Taruishi M, Ozawa K, et al. Diagnosis for early colorectal carcinoma-conventional colonoscopy. Clin Gastroenterol. 2011;26:1589-96 (in Japanese with English Abstract).

4. Kawano H, Tsuruta O, Hasegawa S, et al. Diagnosis of colorectal lesions using conventional endoscopy. Stomach Intest. 2010;45:801-9 (in Japanese with English Abstract).

5. Park W, Kim B, Park SJ, et al. Conventional endoscopic features are not sufficient to differentiate small, early colorectal cancer. World J Gastroenterol. 2014;21:6586-93.

6. Sano Y, Horimatsu T, Fu KI, et al. Magnifying observation of microvascular architecture of colorectal lesions using a narrow band imaging system. Dig Endosc. 2006;18:S44-51.

7. Hirata M, Tanaka S, Oka S, et al. Magnifying endoscopy with narrow band imaging for diagnosis of colorectal tumors. Gastrointest Endosc. 2007;65:988-95.

8. Wada Y, Kudo S, Kashida H, et al. Diagnosis of colorectal lesions with the magnifying narrow-band imaging system. Gastrointest Endosc. 2009;70:522-31.

9. Saito $\mathrm{S}$, Tajiri $\mathrm{H}$, Ohya $\mathrm{T}$, et al. Imaging by magnifying endoscopy with NBI implicates the remnant capillary network as an indication for endoscopic resection in early colon cancer. Int $\mathbf{J}$ Surg Oncol. 2011. doi:10.1155/2011/242608.

10. Hewett DG, Kaltenbach T, Sano Y, et al. Validation of a simple classification system for endoscopic diagnosis of small colorectal polyps using narrow-band imaging. Gastroenterology. 2012;143(3):599-607.

11. Nakayama N, Tanaka S, Sano Y, et al. Validation of the narrow band imaging (NBI) international colorectal endoscopic (NICE) classification for prediction of deep submucosal invasive carcinoma. Gastrointest Endosc. 2012;75:AB338-9.

12. Ikematsu H, Matsuda $T$, Emura $F$, et al. Efficacy of capillary pattern type IIIA/IIIB by magnifying narrow band imaging for estimating depth of invasion of early colorectal neoplasms. BMC Gastroenterol. 2010;10:33.

13. Oka S, Tanaka S, Nakadoi K. Endoscopic features and management of diminutive colorectal submucosal invasive carcinoma. Dig Endosc. 2014;4(26 Suppl 2):78-83.

14. Sakamoto T, Saito Y, Nakajima T, et al. Comparison of magnifying chromoendoscopy and narrow—band imaging in estimation of early colorectal cancer invasion depth : a pilot study. Dig Endosc. 2011;23:118-23.

15. Saito Y, Wada Y, Ikematsu H, et al. Multicenter trial to unify magnified NBI classification using Web test system. Intestine. 2013;17:223-31 (in Japanese with English abstract).

16. Kawasaki K, Kurahara K, Yanai S et al. Significance of a white opaque substance under magnifying narrow-band imaging colonoscopy for the diagnosis of colorectal epithelial neoplasms. Gastrointest Endosc; 2015 (in press).

17. Yamano H, Yoshikawa K, Kimura Y, et al. Precise image diagnosis for colorectal neoplasm magnifying endoscopy observation (pit pattern diagnosis). Stomach Intest. 2010;45:822-8 (in Japanese with English Abstract).

18. Wada Y, Kudo SE, Misawa M, et al. Diagnosis strategy using magnifying endoscopy in early colorectal cancers. Endosc Dig. 2013;25:1204-12 (in Japanese with English abstract).

19. Kawano H, Tsuruta O, Ueno, et al. Diagnosis of early stage gastrointestinal cancer invasion depth via pit pattern observation. Stomach Intest. 2015;50:676-85 (in Japanese with English abstract).

20. Saito $\mathrm{S}$, Aihara $\mathrm{H}$, Tajri $\mathrm{H}$, et al. Autofluorescence imaging makes it easy to differentiate neoplastic lesions from non-neoplastic lesions in the colon. New challenges in gastrointestinal endoscopy. Tokyo: Springer Inc.; 2008. p. 330-7.

21. Aihara H, Sumiyama K, Saito S, et al. Numerous analysis of the autofluorescence intensity of neoplastic and non-neoplastic colorectal lesions by using a novel videoendoscopy system. Gastrointest Endosc. 2009;69:726-33.

22. Inomata $\mathrm{H}$, Tamai $\mathrm{N}$, Aihara $\mathrm{H}$, et al. Efficacy of a novel autofluorescence imaging system with computer-assisted color analysis for assessment of colorectal lesions. World J Gastroenterol. 2013;19(41):7146-53.

23. Ide $\mathrm{D}$, Tamai $\mathrm{N}$, Inomata $\mathrm{H}$, et al. Visualization of colorectal neoplasia by a second-generation autofluorescence imaging system. Scand J Gastroenterol. 2013;48(11):1302-7.

24. Nomura M, Tanuma T, Harada T, et al. Endoscopic ultrasonography in the diagnosis of early colorectal cancer invasion. Stomach Intest. 2015;50:693-702 (in Japanese with English Abstract).

25. Cho E, Miyata M, Nakajima MM. Endoscopic ultrasonography diagnosis for colorectal diseases. New challenges in gastrointestinal endoscopy. Tokyo: Springer Inc.; 2008. p. 338-45.

26. Hamamoto N, Hirata I, Yasumoto S, et al. Diagnosis of the depth of invasion by endoscopic ultrasonography in early colorectal carcinomas. Stomach Intest. 2004;39:1375-86 (in Japanese with English Abstract).

27. Kobayashi K, Kida M, Mukae M, et al. Endoscopic ultrasonography, an accurate imaging technique for the diagnosis of early colorectal cancer. Stomach Intest. 2010;45:811-21 (in Japanese with English Abstract).

28. Saitoh Y, Fujiwara M, Watari J, et al. Invasion depth diagnosis with HFUP and feasibility of indication of endoscopic resection for colorectal submucosal carcinoma. Stomach Intest. 2012;47:491-502 (in Japanese with English Abstract).

29. Saitoh Y, Obara T, Einami K, et al. Efficacy of high-frequency ultrasound probe for the pre-operativestaging of invasion depth in flat and depressed typecolorectal tumors. Gastrointest Endosc. 1996;44:34-9.

30. Santoro GA, Gizzi G, Pellegrini L, et al. The value of highresolution three-dimensional endorectal ultrasonographyin the management of submucosal invasive rectal tumors. Dis Colon Rectum. 2009;52:1837-43. 